\title{
Übereinstimmung von objektiver Messung und subjektiver Erfassung der Arbeitsumgebungsfaktoren Klima, Beleuchtung und Lärm
}

\author{
Maren Formazin ${ }^{1} \cdot$ Martin Schütte ${ }^{1}$ \\ Online publiziert: 17. August 2018 \\ (c) Der/die Autor(en) 2018
}

\section{Zusammenfassung}

Die vorliegende Studie geht der Frage nach, in welchem Maße eine Kongruenz zwischen den Einschätzungen der Umgebungsfaktoren durch Beschäftigte einerseits und den objektiv ermittelten Werten zu Klima, Beleuchtung und Lärm andererseits besteht. Dazu erfolgte die Messung der Ausprägungen der genannten Faktoren in verschiedenen Arbeitsbereichen eines Automobilherstellers mit Hilfe objektiver Verfahren, um darauf basierend Hypothesen über zu erwartende Unterschiede zwischen den Arbeitsbereichen abzuleiten, die auf Basis der Einschätzungen der Merkmale durch Beschäftigte mit Hilfe von varianzanalytischen Verfahren, allgemeinen linearen Modellen sowie zusätzlich mit non-parametrischen statistischen Tests geprüft wurden. Dabei zeigt sich, dass sich Unterschiede in den objektiven Belastungsbedingungen in den Einschätzungen der Beschäftigten zum Teil abbilden: Korrespondenzen bestehen bei Temperatur und Lärm, nicht hingegen bei der Beleuchtung.

Praktische Relevanz: Die Befunde zeigen, dass ein einzelnes Urteil keine zuverlässigen Aussagen über die zwischen den untersuchten Arbeitsbereichen bestehenden Unterschiede erlaubt, sondern Mittelwerte dazu die adäquate Basis darstellen. Weiterhin bestätigt die Untersuchung, dass es für die Ableitung betrieblicher Arbeitsgestaltungsmaßnahmen Items bedarf, die präzise einzelne Aspekte der Arbeitsumgebungsfaktoren erfassen, um Ansatzpunkte für Interventionsmaßnahmen zu identifizieren.

Schlüsselwörter Arbeitsumgebungsfaktor · Objektive Messung $\cdot$ Subjektive Erfassung

\section{Agreement of objective measurement and subjective assessment of the work environment regarding climate, illumination and noise}

\begin{abstract}
The study at hand tests to which degree employees' judgements of work environment factors match objectively measured characteristics on climate, illumination and noise. For this purpose, these work environment factors were recorded in different sections of a plant in the automobile industry via objective measurements to derive hypotheses on which sections were expected to differ in their subjective assessments. These hypotheses were tested based on questionnaire data from employees in the different sections by applying analysis of variance, general linear models and non-parametric test statistics. The results imply that there is congruence between objectively measured and subjectively assessed indicators of temperature and noise whereas discrepancies exist between both kinds of measurements regarding illumination.
\end{abstract}

Dr. Maren Formazin

formazin.maren@baua.bund.de

1 Fachbereich „Arbeit und Gesundheit“,

Bundesanstalt für Arbeitsschutz und Arbeitsmedizin,

Nöldnerstraße 40-42, 10317 Berlin, Deutschland 
Practical Relevance: The empirical results indicate that a single judgement does not allow for reliable statements about differences between the investigated sections. Instead, mean values represent the adequate base for such conclusions. For deriving job design measures, items are required that precisely assess separate aspects of the work environment in order to implement interventions tailored to the existing deficits.

Keywords Work environment $\cdot$ Objective measurement $\cdot$ Subjective assessment

\section{Einführung}

Zur Erhebung der an einem Arbeitsplatz auftretenden Anforderungen existieren sowohl für die betriebliche Praxis als auch für die anwendungsorientierte Forschung unterschiedliche Instrumente, die sich allgemein nach der Art der Datenerhebung (Beobachtung, Befragung), nach dem jeweiligen Anwendungsbereich (Branchen, Organisationsebenen, Berufsgruppen, Tätigkeitsklassen) oder auch nach der theoretischen Fundierung (z.B. Handlungsregulationstheorie) klassifizieren lassen (Dunckel 1999).

Ein etabliertes Verfahren ist die sogenannte „BIBB/ BAuA-Erwerbstätigenbefragung“, ein branchenübergreifend einsetzbares Erhebungsinstrument zur Erfassung von Arbeitsbedingungen, dessen inhaltliche Struktur sich am Belastungs-Beanspruchungs-Konzept orientiert (DIN EN ISO 10075-1 Ergonomische Grundlagen bezüglich psychischer Arbeitsbelastung - Teil 1: Allgemeine Aspekte und Konzepte und Begriffe (ISO 10075-1:2017); Deutsche Fassung EN ISO 10075-1:2017; Rohmert 1972, 1984). Als „Belastung“ werden danach alle von außen auf den Menschen einwirkenden Einflussfaktoren und als „Beanspruchung" die in Abhängigkeit von den jeweiligen individuellen Eigenschaften, Fähigkeiten und Fertigkeiten auftretenden menschbezogenen Belastungswirkungen definiert.

Seit 1979 findet die BIBB/BAuA-Erwerbstätigenbefragung alle fünf bis sechs Jahre statt. 1998/99 war die Bundesanstalt für Arbeitsschutz und Arbeitsmedizin (BAuA) unter der Federführung des Bundesinstituts für Berufsbildung (BIBB) und des Instituts für Arbeitsmarkt- und Berufsforschung (IAB) erstmals an der Erwerbstätigenbefragung beteiligt, die seit 2005/06 kooperativ nur noch vom BIBB und der BAuA durchgeführt wird, wobei jeweils etwa 20.000 Erwerbstätige befragt werden. Wenn auch die Befragung primär darauf zielt, eine Übersicht zu den Arbeitsbedingungen in Deutschland zu gewinnen, so werden ähnlich formulierte Items ebenfalls in betrieblichen Studien, wie etwa der Automobilindustrie, eingesetzt, um Aufschluss über die jeweils gegebene Belastung zu erhalten (Kotzab 2015; Weichel 2012).

Bei der BIBB/BAuA-Erwerbstätigenbefragung schätzen die Befragten im Rahmen eines Telefoninterviews über mehrstufige Ratingskalen die Häufigkeit des Auftretens verschiedener Belastungsfaktoren wie Körperhaltung, physische Arbeit (z.B. Lastenmanipulation), Umgebungsbedingungen (z.B. Lärm, Beleuchtung) sowie psychische Arbeitsanforderungen (Bundesinstitut für Berufsbildung und Bundesanstalt für Arbeitsschutz und Arbeitsmedizin 2012) ein, wobei als Urteilsanker die Häufigkeitsangaben „,häufig“, „,manchmal“, „,selten“ und ,nie“ Verwendung finden. Einem solchen Befragungsansatz liegt allgemein die Annahme zugrunde, dass Beschäftigte valide und reliable Urteile zur Belastung an ihrem Arbeitsplatz abgeben können und auf diese Weise Gestaltungsdefizite identifizierbar sind, denen durch entsprechende Maßnahmen zu begegnen ist.

Allerdings gilt als Nachteil subjektiver Einschätzungen, dass sie verschiedenen Fehlereinflüssen unterliegen können wie z.B. interindividuellen Unterschieden in der Ausnutzung der Bewertungsskala oder persönlichen Einstellungen und Stimmungen (Podsakoff et al. 2003). Weiterhin besteht die Möglichkeit, dass kognitive Verarbeitungsprozesse sowie die Tendenz, sozial erwünscht zu antworten, die subjektiven Urteile mit prägen. Ebenso lässt sich nicht ausschließen, dass in den Einschätzungen eher ein Globalurteil und nicht die Bewertung der jeweils erfragten Faktoren zum Ausdruck kommt. Darüber hinaus bleibt zu berücksichtigen, dass die subjektiven Angaben sich nicht auf die aktuell gegebenen Bedingungen, sondern einen längeren Zeitraum beziehen können (Vischer und Fischer 2005).

So stellt auch Frieling (2014) fest, dass in Beschäftigtenurteile zur ergonomischen Arbeitsgestaltung vermutlich weitere Merkmale der Arbeit wie der Tätigkeitsinhalt eingehen, wodurch es zu Abweichungen zwischen objektiven Messergebnissen und subjektiven Befragungsdaten kommen kann. Dies zeigt sich zum Beispiel in einer in der Automobilindustrie durchgeführten Längsschnittuntersuchung zur Wirkung von Arbeitsgestaltungsmaßnahmen: Objektiv messbare Veränderungen in den Fertigungslinien korrespondierten in vielen Fällen nicht mit entsprechenden Verschiebungen in der subjektiv wahrgenommen Belastung, da in das Urteil der Beschäftigten weitere Merkmale der Arbeit wie psychosoziale Faktoren eingingen (Kotzab 2015).

Besteht das primäre Ziel der BIBB/BAuA Erwerbstätigenbefragung auch nicht in der Ableitung von spezifischen Arbeitsgestaltungsmaßnahmen, muss dennoch der Nachweis erbracht werden, dass die subjektiven Einschätzungen der Befragten die tatsächlich objektiv gegebenen Arbeitsbe- 
dingungen abbilden, um belastungsbezogene Aussagen zu rechtfertigen.

Dabei sind methodisch zwei Vorgehensweisen denkbar: Einmal lässt sich prüfen, in welchem Maße die Einschätzungen der Beschäftigten mit denen von arbeitswissenschaftlichen bzw. betrieblichen Experten korrespondieren. Zum anderen kann bestimmt werden, ob die subjektiven Urteile zwischen objektiv gegebenen Unterschieden in den Arbeitsbedingungen zuverlässig und erwartungskonform zu differenzieren erlauben.

Für die BIBB/BAuA Erwerbstätigenbefragung existiert eine laborexperimentelle Untersuchung zum Bereich Körperhaltungen (Philipp und Schütte 2014). Diese belegt, dass sich eine geringe versus hohe Anzahl von Hockvorgängen, die während der Aufgabenbearbeitung durchzuführen sind, über subjektive Häufigkeitseinschätzungen zuverlässig voneinander differenzieren lässt. In der hier vorliegenden Studie soll die bedingungsbezogene Reliabilität der BIBB/BAuA-Befragung für die Arbeitsumgebungsfaktoren Klima, Beleuchtung und Lärm aufgeklärt werden, um Hinweise darauf zu gewinnen, in welchem Maße eine Kongruenz zwischen den Einschätzungen der Umgebungsfaktoren durch die Beschäftigten einerseits und objektiv ermittelten Werten zu Klima, Beleuchtung und Lärm andererseits besteht.

\section{Methodisches Vorgehen}

\subsection{Objektive Ermittlung der Arbeitsumgebung und Ableitung von Hypothesen}

Die Erhebungen und Messungen zur Beantwortung der Forschungsfrage erfolgten in einem Automobilwerk in vier sich in der Art der durchzuführenden Tätigkeiten unterscheidenden Bereichen: zwei verschiedenen Getriebefertigungen (im folgenden Getriebefertigung A und B), einer Gießerei und einem Presswerk.

Zur Ermittlung der objektiven Kenngrößen Klima, Beleuchtung und Lärm wurden im ersten Quartal 2015 Messungen der Parameter durch Mitarbeitende der BAuA an insgesamt 19 Arbeitsplätzen in den vier Arbeitsbereichen (Getriebefertigung A: sieben Arbeitsplätze; in den übrigen Arbeitsbereichen jeweils vier Arbeitsplätze) unter Berücksichtigung der aktuellen Vorschriften und Normen durchgeführt.

Zur Messung von Lufttemperatur und relativer Luftfeuchte (Messgerät: 176 P1 Testo) wurden Klimasensoren in einer Höhe von $1,10 \mathrm{~m}$ für stehende und $0,60 \mathrm{~m}$ für sitzende Personen in unmittelbarer Nähe des Hauptaufgabenbereiches positioniert. An allen Arbeitsplätzen erfolgten mindestens drei Messungen mit einer Dauer von jeweils $60 \mathrm{~min}$ und mehr zu unterschiedlichen Tageszeiten.
Bei der Erfassung der Beleuchtungsbedingungen erfolgte nach der Festlegung der Hauptsehaufgabe die Ermittlung der Abmessungen der Arbeitsfläche, um anschließend das Messraster zur Bestimmung der Beleuchtungsstärke festlegen zu können, das mindestens neun Messpunkte umfasste. Zur Positionierung des Messgeräts (Gigahertz BTS-256 E bzw. Gigahertz X1) auf der Arbeitsfläche wurde ein Gliedermaßstab oder ein Maßband verwendet. Bei bewegtem oder unebenem Untergrund wurde ein gedachtes Raster abgefahren. Der Farbwiedergabeindex sowie die Farbtemperatur wurden mit dem Optometer Gigahertz X1 mit dem Sensor VL-3701-4 ermittelt.

Zur Erfassung des Schalldruckpegels fanden an allen Arbeitsplätzen mehrere Messungen mit einem Handschallpegelmesser (Brüel \& Kjær, Typ 2250) über eine Dauer von ca. 2 min, alternativ über mehrere Arbeitszyklen, statt.

Diese Befunde bildeten die Grundlagen, auf Basis derer die Ableitung von Hypothesen zu den zu erwartenden Unterschieden in den subjektiven Einschätzungen der Beschäftigten der vier Arbeitsbereiche erfolgte. Diese beziehen sich auf zwei unterschiedliche Detailtiefen: Zum einen wurden Hypothesen über die jeweils allgemeinen Einschätzungen der Faktoren im Sinne eines Globalurteils abgeleitet, zum anderen über die Beurteilung einzelner Teilaspekte des jeweiligen Faktors. Diese Hypothesen wurden anschließend über geeignete statische Analysen auf Grundlage der Befragungsergebnisse gegen den Zufall geprüft.

\subsection{Erhebung der subjektiven Einschätzungen der Arbeitsumgebung}

Zur subjektiven Einschätzung der Arbeitsbedingungen kamen Items aus dem Fragebogen der BIBB/BAuA-Erwerbstätigenbefragung (Bundesinstitut für Berufsbildung und Bundesanstalt für Arbeitsschutz und Arbeitsmedizin 2012) zur physikalischen, physischen und psychischen Belastung zum Einsatz.

Da die Fragen der BIBB/BAuA-Erwerbstätigenbefragung nach den physikalischen Einflussgrößen Klima, Beleuchtung und Lärm, die im Folgenden betrachtet werden, sehr allgemein gestellt sind und keinen differenzierten Rückschluss auf einzelne Teilaspekte des jeweiligen Umgebungsfaktors zulassen, wurden Merkmale der Umgebungsfaktoren Klima und Beleuchtung detaillierter abgefragt. Für den Faktor Lärm lagen keine zusätzlichen Items vor. Eine Übersicht der eingesetzten Items findet sich im Anhang.

Die Befragung der Beschäftigten erfolgte im Rahmen der regelmäßig stattfindenden Teamsitzungen in den vier Arbeitsbereichen im ersten Quartal 2015 bzw. im vierten Quartal 2016. Ein/e geschulte/r TestleiterIn erläuterte den Beschäftigten den Zweck der Untersuchung, die Freiwilligkeit der Teilnahme und die Sicherung der Anonymität der Daten. Nach dieser Einführung wurden die Beschäftig- 
ten gebeten, in den ausgeteilten Fragebögen bei jedem Item jeweils die Ratingkategorie anzukreuzen, die ihre Arbeitssituation am besten beschreibt. Bei Fragen konnten sich die Beschäftigten an den/die TestleiterIn wenden. Die Teilnahme an der Befragung war freiwillig: Wollten Beschäftigte nicht an der Untersuchung teilnehmen, so konnten sie den Fragebogen auch unausgefüllt abgeben.

In einigen wenigen Fällen konnten die Befragungen nicht wie geplant durch den/die TestleiterIn durchgeführt werden, wenn sich z. B. kurzfristig Probleme bei der Produktion ergeben hatten. In diesen Fällen erfolgte das Ausfüllen der Fragebögen in einer späteren Teamsitzung unter Anleitung des jeweiligen Schichtmeisters ohne Beteiligung eines/r Testleiters/In.

\subsection{Klima - Objektive Messergebnisse und Hypothesen}

\subsubsection{Temperatur}

Gemäß der objektiven Messungen liegen die Lufttemperaturen in den beiden Getriebefertigungen $\mathrm{A}$ und $\mathrm{B}$ zwischen $+19,1^{\circ} \mathrm{C}$ und $+21,9^{\circ} \mathrm{C}$, im Presswerk zwischen $+21,6^{\circ} \mathrm{C}$ und $+23,3^{\circ} \mathrm{C}$. Damit unterschreiten alle Werte nicht die nach ASR A3.5 (Ausschuss für Arbeitsstätten 2017) geforderten Mindestwerte für leichte, mittlere bzw. schwere, im Gehen oder Stehen durchzuführende Tätigkeiten von $+19^{\circ} \mathrm{C},+17^{\circ} \mathrm{C}$ bzw. $+12^{\circ} \mathrm{C}$. Weiterhin liegen die Messergebnisse auch nicht über dem für Arbeitsräume geltenden Maximalwert von $+26^{\circ} \mathrm{C}$.

Dagegen nimmt die Lufttemperatur in der Gießerei an zwei von insgesamt vier Arbeitsplätzen durchgängig Werte von mehr als $+26^{\circ} \mathrm{C}$ an und an einem dritten Arbeitsplatz überschreitet sie zumindest zeitweise den Maximalwert von $+26^{\circ} \mathrm{C}$. An einem Arbeitsplatz bleiben die Werte im zulässigen Bereich.

\subsubsection{Relative Luftfeuchte}

Die relative Luftfeuchtigkeit liegt in allen vier Arbeitsbereichen zwischen 17,8 und 34,8\% und überschreitet damit nicht die maximal zulässige Höhe von $80 \%$ bei $+20^{\circ} \mathrm{C}$, $70 \%$ bei $+22^{\circ} \mathrm{C}, 62 \%$ bei $+24^{\circ} \mathrm{C}$ oder $55 \%$ bei $+26^{\circ} \mathrm{C}$ (Ausschuss für Arbeitsstätten 2017).

Aus diesen Befunden lassen sich für die subjektiven Klimabewertungen die nachfolgenden drei Hypothesen ableiten:

Hypothese 1 Die allgemeine Einschätzung des Klimas in der Gießerei unterscheidet sich von der Einschätzung in den drei anderen Bereichen, welche nicht voneinander divergieren. Dabei wird das Klima in der Gießerei ungünstiger eingeschätzt als in den drei anderen Arbeitsbereichen.
Hypothese 2a Die Einschätzung der Temperatur in der GieBerei differiert von der Einschätzung in den drei anderen Bereichen, welche sich nicht voneinander unterscheiden. Dabei wird die Temperatur in der Gießerei ungünstiger eingeschätzt im Sinne von ,zu warm“ als in den drei anderen Arbeitsbereichen.

Hypothese 2b Die Einschätzungen der Feuchtigkeit bzw. Nässe in den vier Arbeitsbereichen weichen nicht voneinander ab.

\subsection{Beleuchtung - Objektive Messergebnisse und Hypothesen}

\subsubsection{Beleuchtungsstärke}

Die Mindestwerte der Beleuchtungsstärke werden in der ASR A3.4 (Ausschuss für Arbeitsstätten 2014) in Abhängigkeit von Arbeitsraum, Arbeitsplatz und von der Tätigkeit angegeben. Sie sollen für grobe Montagearbeiten in der Metallbe- und -verarbeitung 2001x, für mittelfeine Montagearbeiten 3001x, für feine Montagearbeiten 5001x, für sehr feine Montagearbeiten 7501x und in einer Druckgießerei $3001 x$ betragen.

In der Getriebefertigung A erreicht die mittlere Beleuchtungsstärke an zwei von sieben Arbeitsplätzen nicht die nach der ASR A3.4 geforderten Mindestbeleuchtungsstärken.

In der Getriebefertigung B und im Presswerk entsprechen dagegen die mittleren Beleuchtungsstärken aller Arbeitsplätze den vorgegebenen Mindestwerten.

In der Gießerei wird die Mindestbeleuchtungsstärke an drei von vier Arbeitsplätzen unterschritten.

\subsubsection{Gleichmäßigkeit}

Die Gleichmäßigkeit g1 der Beleuchtung hat gemäß ASR A3.4 einen Wert nicht unterhalb von 0,6 anzunehmen (Ausschuss für Arbeitsstätten 2014).

In der Getriebefertigung A entspricht die Gleichmäßigkeit an zwei von sieben Arbeitsplätzen nicht dieser Forderung.

In der Getriebefertigung B bleibt die Gleichmäßigkeit an drei von vier Arbeitsplätzen dann unterhalb des Mindestwertes, wenn die hier zusätzlich vorhandenen Leuchtmittel eingeschaltet sind.

Im Presswerk überschreitet die Gleichmäßigkeit an allen Arbeitsplätzen den verlangten Mindestwert.

In der Gießerei gibt es einen unter den vier Arbeitsplätzen, bei dem die Gleichmäßigkeit den ASR-Vorgaben nicht genügt. 


\subsubsection{Farbwiedergabe}

Nach der ASR A3.4 soll der Farbwiedergabeindex Ra numerisch möglichst nahe 100 liegen und bei Montagearbeiten in der Metallbe- und -verarbeitung den Wert von 80 und in Druckgießereien und im Metallguss den Wert von 60 nicht unterschreiten (Ausschuss für Arbeitsstätten 2014). Die Messungen belegen, dass ausschließlich in der Gießerei sämtliche Arbeitsplätze den Anforderungen an die Farbwiedergabe entsprechen.

Für die Farbtemperatur, gemessen in Kelvin, gibt es keine Mindest- oder Maximalwerte. Je höher die Farbtemperatur, desto kälter die Lichtfarbe. Hier sind die Werte in der Gießerei am niedrigsten (d.h. am wärmsten) und im Presswerk am höchsten (d.h. am kältesten).

Aus diesen Messergebnissen lassen sich für die subjektiven Beleuchtungsbewertungen die nachfolgenden drei Hypothesen ableiten:

Hypothese 3 Die allgemeinen Einschätzungen der Beleuchtung differieren zwischen den vier Arbeitsbereichen. Dabei sollte die Einschätzung in der Gießerei am ungünstigsten und die im Presswerk am günstigsten sein.

Hypothese 4a Die Einschätzung der Beleuchtungsstärke für die Arbeitsfläche und den Raum unterscheidet sich zwischen den Arbeitsbereichen und wird in der Gießerei am ungünstigsten im Sinne von ,zu dunkel“ und in der Getriebefertigung B sowie dem Presswerk am günstigsten (im Sinne von ,genau richtig“) beurteilt.

Hypothese 4b Die Einschätzungen der Lichtfarbe divergieren zwischen den Arbeitsbereichen. In der Gießerei wird die Farbtemperatur eher als warm eingeschätzt und im Presswerk eher als kalt.

\subsection{Lärm - Objektive Messergebnisse und Hypothesen}

Gemäß der „Verordnung zum Schutz der Beschäftigten vor Gefährdungen durch Lärm und Vibrationen“ (LärmVibrationsArbSchV; Bundesministerium der Justiz und für Verbraucherschutz 2017) liegen die Auslösewerte in Bezug auf den Tages-Lärmexpositionspegel und den Spitzenschalldruckpegel bei:

- obere Auslösewerte: L (tief) $\mathrm{EX}, 8 \mathrm{~h}=85 \mathrm{~dB}(\mathrm{~A})$ beziehungsweise L (tief) $p C$, peak $=137 \mathrm{~dB}(\mathrm{C})$ und

- untere Auslösewerte: L (tief) $\mathrm{EX}, 8 \mathrm{~h}=80 \mathrm{~dB}(\mathrm{~A})$ beziehungsweise $\mathrm{L}$ (tief) $\mathrm{pC}$,peak $=135 \mathrm{~dB}(\mathrm{C})$.

Wenn auch nicht direkt übertragbar, so erscheint es doch hilfreich, im Folgenden diese Schwellen als Orientierungs- hilfe für die Einstufung der erhobenen energieäquivalenten Mittelungspegel und Peakwerte zu verwenden.

\subsubsection{Energieäquivalente Mittelungspegel $\left(\mathrm{L}_{\text {Aeq }}\right)$}

In der Getriebefertigung $\mathrm{A}$ erreicht der $\mathrm{L}_{\mathrm{Aeq}}$ an einem von sieben Arbeitsplätzen einen Wert von über $85 \mathrm{~dB}(\mathrm{~A})$.

In der Getriebefertigung B liegt der Schalldruckpegel bei allen vier Arbeitsplätzen unter $80 \mathrm{~dB}(\mathrm{~A})$.

Im Presswerk überschreitet der Schalldruckpegel an einem Arbeitsplatz $85 \mathrm{~dB}(\mathrm{~A})$ sowie an zwei weiteren $\mathrm{Ar}$ beitsplätzen $80 \mathrm{~dB}(\mathrm{~A})$. Beim vierten Arbeitsplatz ergibt sich ein Wert von etwa $80 \mathrm{~dB}(\mathrm{~A})$.

In der Gießerei beträgt der Schalldruckpegel an allen Arbeitsplätzen mehr als $85 \mathrm{~dB}(\mathrm{~A})$.

\subsubsection{Peakwerte $\left(\mathrm{L}_{(\text {peak }}\right)$}

In der Gießerei gehen die Peak-Werte an zwei Arbeitsplätzen über den unteren Auslösewert hinaus. In allen anderen Arbeitsbereichen wird der Peak-Wert an keinem Arbeitsplatz übertroffen.

Hypothese 5 Hinsichtlich der Einschätzung des Lärms unterscheiden sich die vier Arbeitsbereiche voneinander. Die Einschätzung des Lärms wird in der Gießerei und im Presswerk am ungünstigsten sein und in der Getriebefertigung $B$ am günstigsten.

\subsection{Datenanalyse}

Von der Analyse der Daten ausgeschlossen wurden die Beschäftigten, die den Fragenbogen komplett nicht beantwortet hatten bzw. die im Produktionsbereich untypische Tätigkeiten erledigten.

Berücksichtigung fanden zum einen die drei Items aus der BIBB/BAuA-Erwerbstätigenbefragung (Items 1, 2 und 3), die sich auf die Umgebungsfaktoren Klima, Beleuchtung und Lärm beziehen und ein Globalurteil von den Beschäftigten erfordern, und zum anderen die vier zusätzlichen Items zu Teilaspekten des Raumklimas (Items 11, 12, 13 und 14) sowie die drei zusätzlichen Items zu Teilaspekten der Beleuchtung (Items 21, 22 und 23).

In einem ersten Schritt wurde auf Basis des ShapiroWilks-Tests (SW) bzw. des Kolmogorov-Smirnoff-Tests (KS) ermittelt, ob die Daten in den vier Arbeitsbereichen bei einem Alpha-Niveau von $\alpha=0,10$ jeweils normalverteilt sind. Während für die Prüfung der Nullhypothese der Normalverteilung bei Stichproben mit einem $n \leq 50$ der SW angezeigt ist, wird der KS für die Prüfung bei größeren Stichproben empfohlen (Brosius 2004).

Anschließend erfolgte für alle zehn Items jeweils die Ermittlung des Intra-Klassen-Korrelationskoeffizienten 
ICC $(1,1)$ (Shrout und Fleiss 1979), der den auf den Faktor Arbeitsbereich zurückgehenden Anteil an der Gesamtvarianz wiedergibt.

Im nächsten Schritt wurden mit Hilfe einfaktorieller varianzanalytischer Verfahren die Hypothesen 1, 3 und $5 \mathrm{zu}$ Unterschieden zwischen den Arbeitsbereichen jeweils separat für die entsprechenden BIBB/BAuA-Items geprüft, die von den Beschäftigten eine allgemeine Einschätzung (d.h. ein Globalurteil) verlangen. Dies betrifft im Einzelnen folgende drei Items:

- Item 1 zur Erfassung des Klimas,

- Item 2 zur Erfassung der Beleuchtung sowie

- Item 3 zur Erfassung des Lärms.

Bei Vorliegen eines signifikanten Testergebnisses wurde über Post-hoc-Analysen ermittelt, welche Arbeitsbereiche sich unterschieden. Sofern Varianzgleichheit in den Arbeitsbereichen gegeben war, geprüft über den Levene-Test (Brosius 2004), wurde dazu Hochbergs GT2-Verfahren verwendet; bei ungleichen Varianzen die Games-Howell-Prozedur (Field 2009). Um eine Inflation des Fehlers 1. Art zu begrenzen, wurde für das festgelegte Alpha-Niveau von $\alpha=0,05$ auf Basis von sechs post-hoc Vergleichen eine Bonferroni-Korrektur vorgenommen, die zu einem adjustierten $\alpha_{\text {adj }}=0,008$ führte (Field 2009).

Für die beiden Faktoren Klima und Beleuchtung ließ sich bezogen auf die Zusatzitems ermitteln, welche Aspekte des jeweiligen Umgebungsfaktors zwischen den Arbeitsbereichen differenzieren (Prüfung der Hypothesen 2a, 2b, 4a und 4b). Dazu wurden allgemeine lineare Modelle pro Faktor mit listenweisem Fallausschluss bei unvollständigen Daten genutzt. Ein solcher multivariater Ansatz hat den Vorteil, den Abhängigkeiten der einzelnen Aspekte Rechnung zu tragen und wiederum eine Inflation des Fehlers 1. Art einzuschränken. Auch hier wurden zur Ermittlung der sich unterscheidendenden Arbeitsbereiche Post-hoc-Analysen mit Bonferroni-Korrektur des Alpha-Niveaus durchgeführt, wiederum mit dem Hochberg GT2-Verfahren bei Varianzgleichheit in den Arbeitsbereichen und der Games-Howell-Prozedur bei Heterogenität der Varianzen.

Wenngleich varianzanalytische Verfahren insbesondere bei größeren Stichproben $\left(n_{i}>10\right)$ recht robust gegenüber der Verletzung der Annahme normalverteilter Daten sind (Bortz 2005), wurden alle Hypothesen auf Basis des nonparametrischen Kruskal-Wallis-Tests (Field 2009) sowie des Dunn-Bonferroni-Tests für Post-hoc-Analysen (Universität Zürich 2016) jeweils mit Bonferroni-korrigiertem $\alpha=0,05$ (d.h. $\alpha_{\text {adj }}=0,008$ ) zusätzlich geprüft, um im Falle signifikanter Abweichungen von der Gauss-Verteilung Aufschluss über die Stabilität der Ergebnisse zu erhalten.

\section{Ergebnisse}

An der Befragung nahmen insgesamt 344 Beschäftigte aus den vier Arbeitsbereichen teil, von denen die Daten von 340 Beschäftigten in die weitere Analyse eingingen. 83 der Befragten gaben an, jünger als 30 Jahre zu sein, 255 ordneten sich der Altersgruppe der ab 30-Jährigen zu, 2 gaben kein Alter an. Weitere soziodemographische Daten zur Beschreibung der Beschäftigten, etwa zur Dauer der Betriebszugehörigkeit oder der Tätigkeit am Arbeitsplatz, ließen sich aus Gründen des Datenschutzes nicht erheben, da sicherzustellen war, dass kein Rückschluss auf einzelne Beschäftigte möglich ist.

In allen vier Arbeitsbereichen weichen die Daten substantiell $(\alpha \leq 0,10)$ von der Normalverteilung ab.

\subsection{Klima}

\subsubsection{Hypothese 1 - Klima allgemein}

Für das Item 1 (,unter Kälte, Hitze, Nässe, Feuchtigkeit oder Zugluft arbeiten“) ergibt sich für den Faktor „Arbeitsbereich“ eine Varianzkomponente von 0,246 und für die Residualvarianz eine Komponente von 0,751. Demnach lässt sich ein Anteil von 24,7\% der in den Daten vorhandenen Variabilität auf Unterschiede zwischen den Arbeitsbereichen zurückführen.

In der folgenden Tab. 1 sind zu Item 1 die Mittelwerte, Standardabweichungen und die Anzahl der Befragten in den vier Arbeitsbereichen dargestellt.

Das Ergebnis der Varianzanalyse weist mit einem $F(3,269)=27,367 ; p<0,001$ darauf hin, dass sich die vier Arbeitsbereiche signifikant voneinander unterscheiden. Im Ergebnis der Games-Howell-Prozedur wird deutlich, dass die Beschäftigten in der Gießerei in stärkerem Maße zustimmen, unter ungünstigen klimatischen Bedingungen tätig zu sein, als die Beschäftigten in den drei anderen Arbeitsbereichen, welche sich nicht voneinander unterscheiden. Dieses Ergebnis lässt sich über den KruskalWallis-Test sowie die Dunn-Bonferroni-Tests replizieren $(p<0,001)$.

Demzufolge kann die Hypothese 1 angenommen werden.

\subsubsection{Hypothesen $2 a$ und $2 b-$ Klima spezifisch}

In der folgenden Tab. 2 sind die geschätzten Varianzkomponenten für die vier Zusatzitems zum Faktor Klima dargestellt. Der Arbeitsbereich klärt bei den Items 11 „Arbeiten unter Hitze“ und 12 „Temperatur“ jeweils rund 1/3 der Varianz auf, auch beim Item 14 „Arbeiten unter Nässe“ wird durch den Arbeitsbereich circa $1 / 4$ der Varianz aufgeklärt. Dies trifft in deutlich geringerem Maße auf das Item zur 
Tab. 1 Mittelwerte, Standardabweichungen und Stichprobengrößen zu Item 1 (,unter Kälte, Hitze, Nässe, Feuchtigkeit oder Zugluft arbeiten“) in den vier Arbeitsbereichen

Tab. 1 Means, standard deviations and sample sizes for item 1 (climate) in the four sections

\begin{tabular}{|c|c|c|c|c|c|c|c|c|c|c|c|c|c|}
\hline \multirow[t]{2}{*}{ Item } & \multicolumn{3}{|c|}{ Presswerk } & \multicolumn{3}{|c|}{ Getriebe A } & \multicolumn{3}{|c|}{ Getriebe B } & \multicolumn{3}{|c|}{ Gießerei } & \multirow[t]{2}{*}{ Range } \\
\hline & $N$ & $M$ & $S D$ & $N$ & $M$ & $S D$ & $N$ & $M$ & $S D$ & $N$ & $M$ & $S D$ & \\
\hline 1 & 35 & 2,17 & 0,891 & 95 & 2,13 & 1,034 & 41 & 2,34 & 1,087 & 102 & 1,22 & 0,519 & 1 bis 4 \\
\hline
\end{tabular}

Bei der Kodierung in den Daten entspricht der Wert „1“ „,häufig“ und der Wert „4“ „,nie“

Tab. 2 Ergebnisse der Varianzkomponentenanalyse für die vier Zusatzitems zum Faktor Klima

Tab. 2 Results of variance component analysis for the four additional items assessing climate

\begin{tabular}{|c|c|c|c|c|}
\hline Item & & $\begin{array}{l}\text { Varianzkomponen- } \\
\text { tenschätzung } \\
\text { Arbeitsbereich }\end{array}$ & $\begin{array}{l}\text { Varianzkomponen- } \\
\text { tenschätzung } \\
\text { Residualvarianz }\end{array}$ & $\begin{array}{l}\text { Anteil (in \%) der } \\
\text { durch den Arbeits- } \\
\text { bereich aufgeklärten } \\
\text { Varianz }\end{array}$ \\
\hline$\overline{11}$ & Unter Hitze arbeiten. Wie häufig kommt dies vor? & 0,184 & 0,399 & 31,6 \\
\hline 12 & $\begin{array}{l}\text { Ich finde die Temperatur insgesamt ... [7-stufig von „zu kühl“ } \\
\text { über ,,angenehm“ bis „,zu warm“] }\end{array}$ & 0,790 & 1,617 & 32,8 \\
\hline 13 & $\begin{array}{l}\text { Ich finde die Luftfeuchtigkeit insgesamt ... [7-stufig von „,zu } \\
\text { trocken“ über ,,angenehm“ bis „,zu feucht“] }\end{array}$ & 0,114 & 2,052 & 5,2 \\
\hline 14 & Unter Nässe arbeiten. Wie häufig kommt dies vor? & 0,193 & 0,547 & 26,0 \\
\hline
\end{tabular}

Tab. 3 Mittelwerte, Standardabweichungen und Stichprobengrößen zu den vier Zusatzitems zum Klima in den vier Arbeitsbereichen

Tab. 3 Means, standard deviations and sample sizes for the four additional items assessing climate in the four sections

\begin{tabular}{llllllllllrrrr}
\hline Item & \multicolumn{2}{l}{ Presswerk } & \multicolumn{4}{c}{ Getriebe A } & \multicolumn{4}{c}{ Getriebe B } & \multicolumn{3}{c}{ Gießerei } \\
& $N$ & $M$ & $S D$ & $N$ & $M$ & $S D$ & $N$ & $M$ & $S D$ & $N$ & $M$ & $S D$ \\
\hline $\mathbf{1 1}$ & 34 & $\mathbf{1 , 8 8}$ & 0,591 & 93 & $\mathbf{2 , 1 3}$ & 0,837 & 41 & $\mathbf{1 , 7 3}$ & 0,708 & 101 & $\mathbf{1 , 1 1}$ & 0,313 \\
$\mathbf{1 2}$ & 34 & $\mathbf{4 , 1 5}$ & 1,329 & 86 & $\mathbf{4 , 0 0}$ & 1,302 & 39 & $\mathbf{4 , 9 7}$ & 1,460 & 98 & $\mathbf{5 , 9 6}$ & 1,139 & 1 bis 4 \\
$\mathbf{1 3}$ & 35 & $\mathbf{3 , 4 6}$ & 1,197 & 92 & $\mathbf{3 , 5 9}$ & 1,029 & 40 & $\mathbf{3 , 6 5}$ & 1,350 & 99 & $\mathbf{4 , 3 2}$ & 1,812 & 1 bis 7 \\
$\mathbf{1 4}$ & 35 & $\mathbf{3 , 4 9}$ & 0,818 & 93 & $\mathbf{3 , 7 8}$ & 0,529 & 41 & $\mathbf{3 , 9 0}$ & 0,300 & 100 & $\mathbf{2 , 9 0}$ & 0,969 & 1 bis 4 \\
\hline
\end{tabular}

Bei der Kodierung in den Daten zu Item 11 und Item 14 entspricht der Wert „,“ „,häufig“ und der Wert „4“ „,nie“. Bei Item 12 entspricht der Wert „1“ „,zu kühl“, der Wert ,4“ ,,angenehm“ und der Wert „7“ „,Zu warm“. Bei Item 13 entspricht der Wert „1“ „,zu trocken“, der Wert , ,“ ,angenehm“ und der Wert „7““,zu feucht““

Luftfeuchtigkeit (Item 13) zu, bei dem etwa 5\% der Varianz auf den Arbeitsbereich zurückgehen.

In der folgenden Tab. 3 sind zu den vier Zusatzitems zum Klima die Mittelwerte, Standardabweichungen und die Anzahl der Befragten in den vier Arbeitsbereichen dargestellt.

Im multivariaten Test mit dem allgemeinen linearen Modell über alle vier Zusatzitems zum Klima wird über PillaiSpur ein $F$-Wert von $F(12,744)=15,384 ; p<0,001$ ermittelt. Auf dieser Grundlage wurde mit Hilfe der Post-hocTests bestimmt, welche Arbeitsbereiche voneinander divergieren.

Bei den Einschätzungen zum Arbeiten unter Hitze (Item 11) unterscheidet sich die Gießerei nach GamesHowell hypothesenkonform (Hypothese 2a) von allen drei anderen Arbeitsbereichen $(p<0,001)$. Bei der Beurteilung der Temperatur insgesamt (Item 12) weicht die Gießerei von den drei anderen Arbeitsbereichen nach Hochbergs GT2 wie erwartet ab $(p<0,001)$. Allerdings differiert entgegen der Hypothese $2 \mathrm{a}$ - zusätzlich auch die Getriebefertigung $\mathrm{B}$ von der Getriebefertigung A überzufällig $(p=0,001)$. Die non-parametrische Auswertung führt bei beiden Items zum selben Ergebnis.

Wenngleich für die Einschätzung der Luftfeuchtigkeit (Item 13) nur ein relativ geringer Anteil der Varianz durch die Arbeitsbereiche aufgeklärt werden kann, wird diese gemäß Games-Howell von den Beschäftigten in der Gießerei als feuchter eingeschätzt als im Presswerk $(p=0,002)$ und in der Getriebefertigung A $(p=0,004)$. Obwohl die objektiven Messungen nur auf geringe Unterschiede zwischen den Bereichen hinweisen und auch in der Gießerei die gemessenen Werte nicht oberhalb der zulässigen Luftfeuchtigkeit liegen, bewerten die Beschäftigten der Gießerei die Bedingungen in ihrem Arbeitsbereich weniger trocken als die Beschäftigten der beiden anderen Bereiche. Dieses Ergebnis lässt sich über den Kruskal-Wallis-Test sowie die Dunn-Bonferroni-Tests replizieren (Presswerk - Gießerei: $p=0,004$; Getriebefertigung A - Gießerei: $p=0,001$ ).

Dies gilt in ähnlicher Weise für die Aussage zum Arbeiten unter Nässe (Item 14), welches die Beschäftigten in der Gießerei - im Vergleich zu den in den anderen drei Arbeitsbereichen Tätigen - auf Basis von Games-Howell 
Tab. 4 Mittelwerte, Standardabweichungen und Stichprobengrößen zu Item 2 („,bei grellem Licht oder schlechter oder zu schwacher Beleuchtung arbeiten") in den vier Arbeitsbereichen

Tab. 4 Means, standard deviations and sample sizes for item 2 (illumination) in the four sections

\begin{tabular}{|c|c|c|c|c|c|c|c|c|c|c|c|c|c|}
\hline \multirow[t]{2}{*}{ Item } & \multicolumn{3}{|c|}{ Presswerk } & \multicolumn{3}{|c|}{ Getriebe A } & \multicolumn{3}{|c|}{ Getriebe B } & \multicolumn{3}{|c|}{ Gießerei } & \multirow[t]{2}{*}{ Range } \\
\hline & $N$ & $M$ & $S D$ & $N$ & $M$ & $S D$ & $N$ & $M$ & $S D$ & $N$ & $M$ & $S D$ & \\
\hline 2 & 34 & 1,88 & 0,946 & 95 & 2,56 & 0,986 & 41 & 3,05 & 0,999 & 102 & 1,96 & 0,922 & 1 bis 4 \\
\hline
\end{tabular}

Bei der Kodierung in den Daten entspricht der Wert „1“ „,häufig“ und der Wert „4““,nie“

als häufiger auftretend bewerten $(p<0,001)$. Die Ergebnisse der non-parametrischen Auswertung entsprechen denen der parametrischen Auswertung $(p<0,001)$.

Auf die Durchführung von Äquivalenztests wurde verzichtet, da die Post-hoc Tests bei den beiden Items 13 und 14 bereits überzufällige Abweichungen zwischen den Arbeitsbereichen belegen.

Damit ist Hypothese 2b, wonach sich die Einschätzungen der Feuchtigkeit bzw. Nässe der vier Arbeitsbereiche nicht unterscheiden, abzulehnen.

\subsection{Beleuchtung}

\subsubsection{Hypothese 3 - Beleuchtung allgemein}

Für das Item 2 (,,bei grellem Licht oder schlechter oder zu schwacher Beleuchtung arbeiten“) beträgt die geschätzte Varianzkomponente für den Arbeitsbereich 0,281 und die für die Restvarianz 0,921. Demnach lässt sich hier ein Anteil von $23,4 \%$ der Gesamtvarianz durch den Arbeitsbereich aufklären.

In der folgenden Tab. 4 sind zu Item 2 die Mittelwerte, Standardabweichungen und die Anzahl der Befragten in den vier Arbeitsbereichen dargestellt.

Aus dem Ergebnis der Varianzanalyse folgt bei $F(3,268)$ $=16,955 ; p<0,001$, dass sich die vier Arbeitsbereiche signifikant voneinander unterscheiden. Im Ergebnis von Hochbergs GT2-Verfahren wird deutlich, dass die Einschätzungen fast aller Arbeitsbereiche voneinander abweichen (Presswerk - Getriebefertigung A: $p=0,003$; Presswerk - Getriebefertigung B: $p<0,001$; Gießerei - Getriebefertigung A: $p<0,001$; Gießerei - Getriebefertigung $\mathrm{B}$ : $p<0,001)$ mit zwei Ausnahmen: die Gießerei und das Presswerk unterscheiden sich nicht überzufällig, ebenso nicht die Getriebefertigung A und die Getriebefertigung B. Dabei ergeben sich für die Getriebefertigung A und B günstigere Einschätzungen als für das Presswerk und die Gießerei.

Dieses Ergebnis lässt sich über den Kruskal-Wallis-Test sowie die Dunn-Bonferroni-Tests replizieren (Presswerk - Getriebefertigung A: $p=0,001$; Presswerk - Getriebefertigung $\mathrm{B}: p<0,001$; Gießerei - Getriebefertigung $\mathrm{A}$ : $p<0,001$; Gießerei - Getriebefertigung B: $p<0,001$ ).
Entgegen der in Hypothese 3 formulierten Annahme besteht hier nicht nur bei der Gießerei, sondern zusätzlich auch beim Presswerk eine als ungünstig beurteilte Beleuchtung.

\subsubsection{Hypothese $4 a$ und $4 b-$ Beleuchtung spezifisch}

Tab. 5 enthält die Ergebnisse der Varianzkomponentenschätzungen für die drei Zusatzitems zum Faktor Beleuchtung. Dabei beträgt der durch den Arbeitsbereich aufgeklärte Varianzanteil $10 \%$ bei Item 22 (,Wie ist die Beleuchtung auf der Arbeitsfläche bezogen auf Ihre Arbeitsaufgabe?“) und $13 \%$ bei Item 23 (,Wie ist die Beleuchtung im gesamten Raum bezogen auf Ihre Arbeitsaufgabe?“). Für das Item 21 zur Lichtfarbe werden etwa $4 \%$ der Varianz durch den Arbeitsbereich aufgeklärt.

Tab. 6 gibt die Mittelwerte, Standardabweichungen und die Anzahl der Befragten in den vier Arbeitsbereichen zu den drei Zusatzitems zur Beleuchtung wider.

Im multivariaten Test mit dem allgemeinen linearen Modell über die drei Zusatzitems zur Beleuchtung resultiert über Pillai-Spur ein F-Wert von $F(9,783)=5,021$; $p<0,001$. Auf dieser Grundlage wurde mit Hilfe von Hochbergs GT2 ermittelt, welche der Arbeitsbereiche sich voneinander unterscheiden.

Die Ergebnisse der Post-hoc-Tests belegen, dass sowohl die Beleuchtung der Arbeitsfläche (Item 22) als auch die Beleuchtung im Raum (Item 23) in der Gießerei substantiell dunkler eingeschätzt werden als in den Getriebefertigungen A (Item 22: $p=0,002$; Item 23: $p=0,001$ ) und $\mathrm{B}$ (Item 22 und Item 23: $p<0,001$ ). Dabei weisen die Einschätzungen in der Gießerei auf eine zu geringe Beleuchtung hin. Die mittleren Ratings in den Getriebefertigungen $\mathrm{A}$ und $\mathrm{B}$ sind am günstigsten.

Über die Dunn-Bonferroni-Tests werden die Unterschiede in den Einschätzungen zwischen Gießerei und den beiden Getriebefertigungen ebenfalls als signifikant ausgewiesen (Item 22 und Item 23: $p \leq 0,001$ ). Zusätzlich indizieren die Tests substantielle Unterschiede zwischen Gießerei und Presswerk in Bezug auf die Beleuchtung der Arbeitsfläche (Item 22: $p=0,005$ ) sowie zwischen Presswerk und Getriebefertigung B bezogen auf die Beleuchtung im Raum (Item 23: $p=0,003$ ). 
Tab. 5 Ergebnisse der Varianzkomponentenanalyse für die drei Zusatzitems zum Faktor Beleuchtung

Tab. 5 Results of variance component analysis for the three additional items assessing illumination

\begin{tabular}{|c|c|c|c|c|}
\hline Item & & $\begin{array}{l}\text { Varianzkomponen- } \\
\text { tenschätzung } \\
\text { Arbeitsbereich }\end{array}$ & $\begin{array}{l}\text { Varianzkomponen- } \\
\text { tenschätzung } \\
\text { Residualvarianz }\end{array}$ & $\begin{array}{l}\text { Anteil (in \%) } \\
\text { der durch den } \\
\text { Arbeitsbereich } \\
\text { aufgeklärten } \\
\text { Varianz } \\
\end{array}$ \\
\hline 21 & Wie empfinden Sie die Lichtfarbe? [7-stufig von „kalt“ bis „warm“] & 0,070 & 1,754 & 3,8 \\
\hline 22 & $\begin{array}{l}\text { Wie ist die Beleuchtung auf der Arbeitsfläche bezogen auf Ihre } \\
\text { Arbeitsaufgaben? [7-stufig von ,zu dunkel“ über „,genau richtig“ zu } \\
\text { „zu hell“] }\end{array}$ & 0,214 & 1,889 & 10,2 \\
\hline 23 & $\begin{array}{l}\text { Wie ist die Beleuchtung im gesamten Raum bezogen auf Ihre Ar- } \\
\text { beitsaufgaben? [7-stufig von ,zu dunkel“ über ,genau richtig“ zu } \\
\text { „Zu hell“] }\end{array}$ & 0,205 & 1,355 & 13,1 \\
\hline
\end{tabular}

Tab. 6 Mittelwerte, Standardabweichungen und Stichprobengrößen zu den drei Zusatzitems zur Beleuchtung in den vier Arbeitsbereichen Tab. 6 Means, standard deviations and sample sizes for the three additional items assessing illumination in the four sections

\begin{tabular}{|c|c|c|c|c|c|c|c|c|c|c|c|c|c|}
\hline \multirow[t]{2}{*}{ Item } & \multicolumn{3}{|c|}{ Presswerk } & \multicolumn{3}{|c|}{ Getriebe A } & \multicolumn{3}{|c|}{ Getriebe B } & \multicolumn{3}{|c|}{ Gießerei } & \multirow[t]{2}{*}{ Range } \\
\hline & $N$ & $M$ & $S D$ & $N$ & $M$ & $S D$ & $N$ & $M$ & $S D$ & $N$ & $M$ & $S D$ & \\
\hline 21 & 34 & 3,09 & 1,485 & 92 & 3,58 & 1,234 & 41 & 3,59 & 1,414 & 100 & 3,86 & 1,311 & 1 bis 7 \\
\hline 22 & 35 & 3,54 & 1,291 & 93 & 3,49 & 1,479 & 40 & 4,03 & 1,121 & 102 & 2,81 & 1,391 & 1 bis 7 \\
\hline 23 & 35 & 3,26 & 1,094 & 94 & 3,51 & 1,216 & 40 & 4,03 & 1,000 & 102 & 2,88 & 1,196 & 1 bis 7 \\
\hline
\end{tabular}

Bei der Kodierung in den Daten zu Item 21 entspricht der Wert „, “ „kalt“ und der Wert „,““ „warm“. Bei den Items 22 und 23 entspricht der Wert „1“ „,zu dunkel“, der Wert „4“" „genau richtig“ und der Wert , ,““, ,zu hell““

Damit ist die Hypothese 4a, wonach die Beleuchtung in der Gießerei am ungünstigsten im Sinne von ,zu dunkel““ und in der Getriebefertigung B sowie dem Presswerk am günstigsten ist, teilweise abzulehnen.

Für die Einschätzung der Lichtfarbe (Item 21) wird im Post-hoc-Test kein substantieller Unterschied zwischen den Arbeitsbereichen ausgewiesen $(p \geq 0,022)$.

Ausgehend von den Dunn-Bonferroni-Tests tritt hier allerdings ein signifikanter Unterschied mit $p=0,001$ in den Einschätzungen zwischen den Beschäftigten in der Gießerei und im Presswerk auf. Da zwischen der parametrischen und der non-parametrischen Auswertung Widersprüche in den Ergebnissen bestehen, lässt sich eine Entscheidung über die Annahme oder Zurückweisung der Hypothese 4b nicht treffen.

\subsection{Lärm}

\subsubsection{Hypothese 5 - Lärm allgemein}

Für das Item 3 (,unter Lärm arbeiten“) nimmt die für den Arbeitsbereich ermittelte Varianzkomponente einen Wert von 0,213 und die für die Residualvarianz einen Wert von 0,409 an. Demnach lässt sich mit 34,3\% hier ein substantieller Anteil der Gesamtvarianz durch den Arbeitsbereich aufklären.

Tab. 7 enthält zu Item 3 die Mittelwerte, Standardabweichungen und die Anzahl der Befragten in den vier Arbeitsbereichen.
Das Ergebnis der Varianzanalyse mit $F(3,267)=45,164$; $p<0,001$ weist auf signifikante Unterschiede zwischen den vier Arbeitsbereichen hin. Im Ergebnis des Games-HowellVerfahrens wird deutlich, dass die Einschätzungen aller Arbeitsbereiche voneinander abweichen $(p \leq 0,006)$ mit Ausnahme der Ratingwerte im Presswerk und der Gießerei.

Die verteilungsfreie Datenauswertung korrespondiert mit diesen Ergebnissen $(p \leq 0,003)$.

Auf Grundlage der objektiven Messungen wurde die ungünstigste Einschätzung in der Gießerei sowie im Presswerk und die günstigste in der Getriebefertigung B erwartet. Demnach ist Hypothese 5 teilweise anzunehmen.

\section{Diskussion und Schlussfolgerungen}

Mit der vorliegenden Untersuchung sollte Aufschluss darüber gewonnen werden, ob sich in der Ausprägung der Arbeitsumgebungsfaktoren Lärm, Klima und Beleuchtung jeweils objektiv bestehende Unterschiede auch in den entsprechenden subjektiven Einschätzungen der Beschäftigten zeigen.

Die Ergebnisse der dazu bei einem Automobilhersteller in vier Arbeitsbereichen durchgeführten Erhebungen demonstrieren insgesamt, dass sich Abweichungen in der physikalischen Arbeitsumgebung, die über die Messung entsprechender Parameter ermittelt wurden, zum Teil ebenfalls auf Basis subjektiver Einschätzungen (Items der BIBB/ BAuA-Erwerbstätigenbefragung, zusätzliche Items zur dif- 
Tab. 7 Mittelwerte, Standardabweichungen und Stichprobengrößen zu Item 3 (,unter Lärm arbeiten“) in den vier Arbeitsbereichen

Tab. 7 Means, standard deviations and sample sizes for item 3 (noise) in the four sections

\begin{tabular}{lllllllllllll}
\hline Item & \multicolumn{2}{l}{ Presswerk } & \multicolumn{4}{c}{ Getriebe A } & \multicolumn{3}{c}{ Getriebe B } & \multicolumn{3}{c}{ Gießerei } \\
& $N$ & $M$ & $S D$ & $N$ & $M$ & $S D$ & $N$ & $M$ & $S D$ & $N$ & $M$ & $S D$ \\
\hline $\mathbf{3}$ & 34 & $\mathbf{1 , 0 6}$ & 0,343 & 94 & $\mathbf{2 , 0 1}$ & 0,956 & 41 & $\mathbf{1 , 4 9}$ & 0,711 & 102 & $\mathbf{1 , 0 0}$ & 0 \\
\hline
\end{tabular}

Bei der Kodierung in den Daten entspricht der Wert „,“ „häufig“ und der Wert „4“ „,nie“

ferenzierten Beurteilung der Arbeitsumgebungsfaktoren) identifizieren lassen.

So bilden sich in den Gesamteinschätzungen des Raumklimas (Item 1) Unterschiede zwischen den Arbeitsbereichen ab, wie sie sich auch in den objektiven Temperaturmessungen zeigen. Das legt nahe, dass in die Beurteilungen vor allem das Kalt-Warm-Empfinden einging. Die Bewertungen, die sich auf das „Arbeiten unter Hitze“ (Item 11) und die „Temperatur“ (Item 12) beziehen, stützen diese Vermutung, da auch hier eine hohe Korrespondenz zwischen objektiven und subjektiven Daten besteht. Dieses Ergebnis ist auch unter physiologischen Aspekten plausibel, da der Mensch für das ,kalt-warm“ Empfinden über Thermorezeptoren in der Haut verfügt. Die zur Feuchtigkeit gewonnenen Einschätzungen zeigen dagegen keine Parallelen zu den entsprechenden Messwerten, da sich hier Unterschiede in den Einschätzungen der Beschäftigten aus den vier Arbeitsbereichen zeigen, die auf Basis der objektiven Messungen nicht erwartet wurden. Möglicherweise ist dies mit dadurch bedingt, dass der Mensch keine Feuchtigkeitsrezeptoren besitzt (Filingeri und Havenith 2015). Wenn auch bei außergewöhnlich feuchten Klimaten durch die fehlende Verdunstung des Schweißes auf der Haut oder eine durchfeuchtete Kleidung ein vor allem haptisch vermittelter Feuchtigkeitseindruck entstehen kann, so scheint eine zuverlässige Bewertung der Feuchtigkeit bei weniger extremem Klima schwerer möglich zu sein (Filingeri und Havenith 2015).

Bei der Beurteilung der Beleuchtung allgemein (Item 2: bei grellem Licht, schlechter oder zu schwacher Beleuchtung arbeiten) finden sich nur teilweise Entsprechungen zwischen den Messergebnissen und den subjektiven Daten. Die Beschäftigten bewerten zwar den objektiv am ungünstigsten beleuchteten Arbeitsbereich - die Gießerei entsprechend negativ, jedoch weisen die Einschätzungen beim Presswerk ebenfalls auf Defizite in der Beleuchtung hin, was den objektiven Messungen allerdings überwiegend widerspricht. Damit erscheint die Schlussfolgerung berechtigt, dass in die generellen Beleuchtungsbewertungen (Item 2) noch andere Aspekte eingegangen zu sein scheinen als nur das Beleuchtungsniveau (siehe dazu auch Frieling 2014; Kotzab 2015). Eine solche Vermutung wird unterstützt durch den Befund, dass sich die Einschätzungen zur Ausleuchtung der Arbeitsfläche (Item 22) beim Presswerk von denen der günstiger beleuchteten Arbeitsplätze in den Getriebefertigungen A und B nicht substantiell unterscheiden und in ihrer absoluten Höhe nahe am Optimal- wert 4 (,genau richtig“) liegen. Äußerungen der Beschäftigten deuten weiterhin darauf hin, dass auftretende Reflexionen oder Blendungen zu den negativen Beurteilungen beim allgemeinen Item 2 geführt haben können, was über zusätzliche Leuchtdichtemessungen objektivierbar gewesen wäre.

Bei den spezifischen Merkmalen der Beleuchtung (Items 21, 22, 23) bestehen Unterschiede in den Ergebnissen der parametrischen und nichtparametrischen Posthoc-Analysen. Dies impliziert, dass die Ergebnisse nicht als stabil angesehen werden können, was einer weitergehenden Interpretation entgegensteht.

In diesem Rahmen bleibt zu bedenken, dass Ältere im Vergleich zu Jüngeren einen höheren Lichtbedarf haben und blendempfindlicher sind (Schierz 2008). Bedingt durch die sehr grobe Altersklassifikation ließ sich das Alter als zusätzliche Variable in die statistischen Analysen jedoch nicht einbeziehen. Daneben ist zu beachten, dass altersbezogene Effekte nicht in die Hypothesen aufgenommen werden konnten, weil in den Technischen Regeln für Arbeitsstätten (Ausschuss für Arbeitsstätten 2014) zwar festgestellt wird, dass ,... mit zunehmendem Alter eine höhere Anforderung an die Beleuchtungsqualität (z.B. eine höhere Beleuchtungsstärke und höhere Anforderungen an die Begrenzung der Blendung) ...“ (S. 5) bestehen kann, die Mindestwerte der Beleuchtungsstärke allerdings nicht nach Alter differenziert angegeben sind. Weiterhin bezieht sich die DIN EN 12464-1 (DIN EN 12464-1:2011-08 Licht und Beleuchtung - Beleuchtung von Arbeitsstätten - Teil 1: Arbeitsstätten in Innenräumen; Deutsche Fassung EN 124641:2011) ebenfalls nur auf Menschen mit normalem Sehvermögen (vgl. hierzu auch Frieling et al. 2012).

Bei der Erhebung der Belastung durch Schall am Arbeitsplatz sind sowohl über die gemessenen Pegel als auch die subjektiven Lärmbeurteilungen übereinstimmend $\mathrm{Ar}$ beitsbereiche mit suboptimalen Bedingungen zu identifizieren. In einer arbeitswissenschaftlichen Untersuchung in Kindertagesstätten (Buch und Frieling 2001, 2002) ließen sich ebenfalls Belastungsspitzen - in Übereinstimmung mit der objektiven Schallbelastung - auf Basis der subjektiven Einschätzungen feststellen, was zu der Schlussfolgerung berechtigt, dass insbesondere hoch belastende Arbeitsplätze auch über subjektive Einschätzungen ermittelbar sind.

Die dagegen in anderen, in unterschiedlichen Industriezweigen durchgeführten Feldstudien ermittelten geringen korrelativen Zusammenhänge zwischen subjektiv und ob- 
jektiv erhobenen Daten (Koradecka et al. 2010) sind vermutlich dadurch bedingt, dass beeinträchtigende Lärmwirkungen (wie z.B. das Erleben von Lästigkeit) auch unterhalb der in der LärmVibrationsArbSchV (Bundesministerium der Justiz und für Verbraucherschutz 2017) aufgeführten, sich an der Gehörschädigung orientierenden Auslösewerte auftreten können (Rothe et al. 2017).

Allgemein fällt bei allen Bewertungen der hohe Residualvarianzanteil auf, der durchgängig mehr als $65 \%$ beträgt, so dass für weitere Aussagen ausschließlich gruppenbezogene Maße der zentralen Tendenz die adäquate Basis darstellen dürften.

Die in der vorliegenden Untersuchung gewonnenen Globaleinschätzungen des Klimas und Lärms (Items der BIBB/BAuA Befragung) sind mehrheitlich hypothesenkonform und geben dementsprechend Hinweise auf die durch die Lufttemperatur und den Schalldruckpegel entstehende Belastung, insbesondere auf eine ungünstige Ausprägung beider Faktoren. Diese Schlussfolgerung wird dadurch gestützt, dass die Ergebnisse der parametrischen und der nonparametrischen Verfahren $\mathrm{zu}$ denselben Entscheidungen über die Annahme bzw. Ablehnung der Hypothesen führen, was die Stabilität der Ergebnisse unterstreicht. Die subjektiven Einschätzungen zur Beleuchtung lassen dagegen keine Aussagen zum Beleuchtungsniveau und der damit einhergehenden Belastung zu.

Eine mögliche Ursache für die unterschiedliche Kongruenz der zu den Arbeitsumgebungsfaktoren erhaltenen objektiven und subjektiven Daten können die jeweiligen Itemformulierungen darstellen. So ist das Item 1 zur allgemeinen Einschätzung des Klimas durch Beispiele konkretisiert, die inhaltlich vor allem mit der Temperatur (Kälte, Hitze, Zugluft) in Beziehung stehen. Hingegen enthält das Item zur Beleuchtung (Item 2) solche Präzisierungen nicht, und der hier zentrale Aspekt ,schlechte bzw. schwache Beleuchtung" eröffnet einen großen Interpretationsspielraum. Hier ist zu prüfen, ob die Identifikation von Mängeln in der Beleuchtungsgestaltung auf Grundlage subjektiver Urteile eher gelingt, wenn insbesondere beleuchtungsbezogene Störeinflüsse wie z.B. auftretende Blendungen oder Reflexionen einzuschätzen sind, setzt doch die Ableitung von Ansatzpunkten zur Arbeitsgestaltung eine präzise, detaillierte Erfassung der einzelnen Arbeitsumgebungsaspekte voraus.

Aufgrund der Datenschutzregelungen war es nicht möglich, eine genaue Zuordnung der subjektiven Bewertungen zu den jeweiligen Arbeitsplätzen vorzunehmen, wie dies in Studien zum Beispiel in einem Krankenhaus (Dianat et al. 2013) oder einer Verpackungsfabrik (Vahedi und Dianat 2014) gelang und in denen höhere Übereinstimmungen zwischen objektiven und subjektiven Beleuchtungsbedingungen berichtet wurden.
Ausgehend von den in der vorliegenden Studie erhaltenen Befunden erscheint es sinnvoll, bei zukünftigen Untersuchungen der Frage nachzugehen, ob subjektive Bewertungsverfahren konsistentere zusätzliche gestaltungsrelevante Informationen liefern, wenn die Beurteilungen weniger auf die quantitative Ausprägung, sondern eher auf qualitative Merkmale der Arbeitsumgebungsfaktoren zielen wie etwa das Lästigkeitserleben bei Lärm oder das Komfortempfinden bei Klima und Beleuchtung.

Danksagung Die Autoren danken Frau Ilka Ahrendt und ihren Mitarbeitenden aus der Gruppe 2.5 Labor „Produkte und Arbeitssysteme“ der Bundesanstalt für Arbeitsschutz und Arbeitsmedizin für die Ermittlung der objektiven Parameter zu Klima, Beleuchtung und Lärm sowie Frau Dr. Gerlinde Kaul aus dem Fachbereich 3 „Arbeit und Gesundheit" der Bundesanstalt für Arbeitsschutz und Arbeitsmedizin für die Aufbereitung der Daten für die vorliegenden Analysen.

\section{Anhang}

\section{Übersicht der in den Analysen berücksichtigten Items}

\section{Klima}

Item 1 unter Kälte, Hitze, Nässe, Feuchtigkeit oder Zugluft arbeiten

Item 11 unter Hitze arbeiten

Item 12 Ich finde die Temperatur insgesamt ... [7-stufig von ,zu kühl“ über ,,angenehm“ bis ,,zu warm“]

Item 13 Ich finde die Luftfeuchtigkeit insgesamt ... [7stufig von ,zu trocken“ über ,angenehm“ bis ,zu feucht"]

Item 14 unter Nässe arbeiten. Wie häufig kommt dies vor?

\section{Beleuchtung}

Item 2 bei grellem Licht oder schlechter oder $\mathrm{zu}$ schwacher Beleuchtung arbeiten

Item 21 Wie empfinden Sie die Lichtfarbe? [7-stufig von ,kalt" bis ,,warm“]

Item 22 Wie ist die Beleuchtung auf der Arbeitsfläche bezogen auf Ihre Arbeitsaufgaben? [7-stufig von ,zu dunkel“ über ,genau richtig“ zu ,,zu hell"]

Item 23 Wie ist die Beleuchtung im gesamten Raum bezogen auf Ihre Arbeitsaufgaben? [7-stufig von „,zu dunkel“ über ,genau richtig“ zu ,,zu hell“"] 


\section{Lärm}

\section{Item 3 unter Lärm arbeiten}

Open Access Dieser Artikel wird unter der Creative Commons Namensnennung 4.0 International Lizenz (http://creativecommons.org/ licenses/by/4.0/deed.de) veröffentlicht, welche die Nutzung, Vervielfältigung, Bearbeitung, Verbreitung und Wiedergabe in jeglichem Medium und Format erlaubt, sofern Sie den/die ursprünglichen Autor(en) und die Quelle ordnungsgemäß nennen, einen Link zur Creative Commons Lizenz beifügen und angeben, ob Änderungen vorgenommen wurden.

\section{Literatur}

Ausschuss für Arbeitsstätten (2014) Technische Regeln für Arbeitsstätten. Beleuchtung. ASR A3.4. https://www.baua.de/DE/Angebote/ Rechtstexte-und-Technische-Regeln/Regelwerk/ASR/pdf/ASRA3-4.pdf?_blob=publicationFile. Zugegriffen: 16. Nov. 2017

Ausschuss für Arbeitsstätten (2017) Technische Regeln für Arbeitsstätten. Raumtemperatur. ASR A3.5. https://www.baua.de/DE/ Angebote/Rechtstexte-und-Technische-Regeln/Regelwerk/ASR/ ASR-A3-5.html. Zugegriffen: 16. Nov. 2017

Bortz J (2005) Statistik für Human- und Sozialwissenschaftler, 6. Aufl. Springer, Heidelberg

Brosius F (2004) SPSS 12. mitp, Bonn

Buch M, Frieling E (2001) Belastungs- und Beanspruchungsoptimierung in Kindertagesstätten. Unfallkasse Hessen, Universität Gesamthochschule Kassel, Kassel

Buch M, Frieling E (2002) Ableitung und Evaluation von Arbeitsgestaltungsmaßnahmen bei ErzieherInnen in Kindertagesstätten. In: Badura B, Litsch M, Vetter C (Hrsg) Fehlzeiten-Report 2001. Springer, Berlin, S 103-118

Bundesinstitut für Berufsbildung, Bundesanstalt für Arbeitsschutz und Arbeitsmedizin (2012) BIBB/BAuA-Erwerbstätigenbefragung 2011/2012. https://www.baua.de/DE/Themen/Arbeitswelt-undArbeitsschutz-im-Wandel/Arbeitsweltberichterstattung/Arbeits bedingungen/pdf/Fragebogen.pdf?_blob=publicationFile\&v=3. Zugegriffen: 16. Nov. 2017

Bundesministerium der Justiz und für Verbraucherschutz (2017) Verordnung zum Schutz der Beschäftigten vor Gefährdungen durch Lärm und Vibrationen. https://www.gesetze-im-internet.de/1_ rmvibrationsarbschv/. Zugegriffen: 16. Nov. 2017 (Lärm- und Vibrations-Arbeitsschutzverordnung - LärmVibrationsArbSchV)

Dianat I, Sedghi A, Bagherzade J, Jafarabadi MA, Stedmon AW (2013) Objective and subjective assessments of lighting in a hospital setting: Implications for health, safety and performance. Ergonomics 56(10): 1535-1545

DIN (2011) EN 12464-1:2011-08 Licht und Beleuchtung - Beleuchtung von Arbeitsstätten - Teil 1: Arbeitsstätten in Innenräumen. Beuth, Berlin (Deutsche Fassung EN 12464-1)

DIN (2017) EN ISO 10075-1 Ergonomische Grundlagen bezüglich psychischer Arbeitsbelastung - Teil 1: Allgemeine Aspekte und Konzepte und Begriffe. Beuth, Berlin (Deutsche Fassung EN ISO 10075-1)

Dunckel H (1999) Handbuch psychologischer Arbeitsanalyseverfahren. vdf Hochschulverlag, Zürich

Field A (2009) Discovering statistics using SPSS, 3. Aufl. SAGE, Los Angeles
Filingeri D, Havenith G (2015) Human skin wetness perception: psychophysical and neurophysiological bases. Temperature 2(1):86-104

Frieling E (2014) Theorie- und Methodendefizite der Arbeitswissenschaft - ein Zwischenruf! Z Arb Wiss 68(4):229-230

Frieling E, Kotzab D, Enriquez-Diaz A, Sytch A (2012) „Mit der Taktzeit am Ende“" - Die älteren Mitarbeiter in der Automobilbranche. Ergonomia, Stuttgart

Koradecka D, Pośniak M, Widerszal-Bazyl M, Augustyńska D, Radkiewicz P (2010) A comparative study of objective and subjective assessment of occupational risk. Int J Occup Saf Ergon $16(1): 3-22$

Kotzab D (2015) Vergleich ergonomischer Bewertungsmethoden mit dem subjektiven Befinden der Mitarbeiter: Eine Längsschnittstudie an Fließbandarbeitsplätzen in der Automobilindustrie. kassel university press, Kassel

Philipp JJ, Schütte M (2014) Zur Einschätzung der Auftretenshäufigkeit von Körperhaltungen. In: Gesellschaft für Arbeitswissenschaften (Hrsg) Gestaltung der Arbeitswelt der Zukunft 60. Arbeitswissenschaftlicher Kongress der GfA, München, 12.-14.03.2014 GfA press, Dortmund, S 531-533

Podsakoff PM, MacKenzie SB, Lee J-Y, Podsakoff NP (2003) Common method biases in behavioral research: A critical review of the literature and recommended remedies. J Appl Psychol 88(5):879-903

Rohmert W (1972) Aufgaben und Inhalt der Arbeitswissenschaft. Berufsb Sch 24(1):3-14

Rohmert W (1984) Das Belastungs-Beanspruchungs-Konzept. Z Arb Wiss 38:193-200

Rothe I, Adolph L, Beermann B, Schütte M, Windel A, Grewer A, Lenhardt U, Michel J, Thomson B, Formazin M (2017) Psychische Gesundheit in der Arbeitswelt. Wissenschaftliche Standortbestimmung. Bundesanstalt für Arbeitsschutz und Arbeitsmedizin, Dortmund, Berlin, Dresden

Schierz C (2008) Licht für die ältere Bevölkerung - physiologische Grundlagen und ihre Konsequenzen. In: Deutsche Lichttechnische Gesellschaft e. V., Lichttechnische Gesellschaft Österreichs, Schweizer Licht Gesellschaft, Nederlandse Stichting voor Verlichtingskunde, Technische Universität Ilmenau (Hrsg) Licht 2008 18. Gemeinschaftstagung, Ilmenau, 10. bis 13. September 2008 LiTG, Berlin, S 32-41 (https://www.db-thueringen. de/servlets/MCRFileNodeServlet/dbt_derivate_00016297/Licht 2008_S.pdf)

Shrout PE, Fleiss JL (1979) Intraclass correlations: Uses in assessing rater reliability. Psychol Bull 86(2):420-428

Universität Zürich (2016) Kruskal-Wallis-Test. http://www.methoden beratung.uzh.ch/de/datenanalyse/unterschiede/zentral/kruskal. html. Zugegriffen: 16. Febr. 2018

Vahedi A, Dianat I (2014) Employees' perception of lighting conditions in manufacturing plants: associations with illuminance measurements. J Res Health Sci 14(1):40-45

Vischer JC, Fischer G-N (2005) Une approche de l'evaluation des environnements de travail: La méthode diagnostique. Trav Hum 68(1):73-95 (User evaluation of the work environment: A diagnostic approach)

Weichel J (2012) Ressourcen und Belastungen in der Berufsbiografie. Eine Studie zum Zusammenhang zwischen berufsbiografischen Arbeitsbedingungen, Gesundheit und Leistungsfähigkeit. kassel university press, Kassel 